\title{
MicroRNAs as Potential Biomarkers in Acute Promyelocytic Leukaemia
}

\author{
Imilia Ismail, ${ }^{1,2}$ Sarina Sulong, ${ }^{3}$ and Rosline Hassan ${ }^{1}$ \\ ${ }^{1}$ Department of Haematology, School of Medical Sciences, Universiti Sains Malaysia, Health Campus, \\ 16150 Kubang Kerian, Kelantan, Malaysia \\ ${ }^{2}$ Faculty of Medicine and Health Sciences, Universiti Sultan Zainal Abidin, 20400 Kuala Terengganu, Terengganu, Malaysia \\ ${ }^{3}$ Human Genome Centre, School of Medical Sciences, Universiti Sains Malaysia, Health Campus, \\ 16150 Kubang Kerian, Kelantan, Malaysia \\ Correspondence should be addressed to Rosline Hassan; roslin@usm.my
}

Received 24 June 2014; Revised 28 October 2014; Accepted 20 November 2014; Published 7 December 2014

Academic Editor: Alfredo Pulvirenti

Copyright (C) 2014 Imilia Ismail et al. This is an open access article distributed under the Creative Commons Attribution License, which permits unrestricted use, distribution, and reproduction in any medium, provided the original work is properly cited.

Acute promyelocytic leukaemia (APL) is an M3 subtype of acute myeloid leukaemia (AML). This classification is based on the morphology of promyelocytic cell. The clinical characteristics of APL can be recognized by haemorrhagic episodes, a differentiation block at the promyelocytic stage, and sensitivity to the differentiation response to all-trans-retinoic acid (ATRA). Cytogenetically, APL is characterized by a balanced reciprocal translocation between chromosomes 15 and 17, which results in the production of $\mathrm{PML} / \mathrm{RAR} \alpha$ fusion protein. Recent studies reported that microRNAs (miRNAs) have also been proposed to contribute to the pathogenesis of APL. miRNAs have been associated with the pathogenesis of cancer and their involvement as oncogenic and tumour suppressor activities have been identified. They are involved in various biological processes including the cell proliferation, differentiation, growth and development, metabolism, apoptosis, and haematopoiesis. The new discovery of miRNAs as possible therapeutic markers will provide new insight for the diagnosis and therapeutic entries for the treatment of APL. This review highlights the potential of miRNAs as biomarkers in APL.

\section{Introduction}

Acute promyelocytic leukaemia (APL) is identified as the M3 subtype of acute myeloid leukaemia (AML) by the FrenchAmerican-British (FAB) classification. This classification is based on the percentage of maturing cells beyond the myeloblast stage. Bone marrow shows hypercellular promyelocytes and the most striking feature of these cells is the cytoplasmic hypergranularity. Multiple Auer rods are also observed in a few early cells in APL. According to WHO 2008 classification, APL is characterized by a reciprocal translocation between chromosomes 15 and 17, which results in the fusion between the promyelocytic leukaemia $(P M L)$ gene and retinoic acid receptor $\alpha(R A R \alpha)$ gene [1]. A schematic representation of the chromosomes and genes involved in $t(15 ; 17)$ is shown in Figure 1.

The breakpoints on chromosome 17 are consistently located within the second intron of the RAR $\alpha$ gene, but, on chromosome 15, there are different breakpoint cluster regions, namely $b c r 1, b c r 2$, and $b c r 3$ located in intron 6 , exon 6 , and intron 3 , respectively, of the $P M L$ gene $[2,3]$. The location of $b c r 1, b c r 2$, and $b c r 3$ produces fusion transcripts of varying lengths referred to as the long, variant, and short forms, respectively [3]. This translocation can be detected by karyotyping or fluorescence in situ hybridization (FISH) studies, and the transcript can be detected by molecular techniques like the polymerase chain reaction (PCR) technique.

Approximately $40 \%$ to $55 \%$ of $t(15 ; 17)$ positive cases were identified within breakpoint region, referred to as $b c r 1$ followed by $b c r 3$ in approximately $37 \%$ to $45 \%$ cases. The most uncommon breakpoint region, $b c r 2$, is involved in roughly $8 \%$ to $14 \%$ of $\mathrm{t}(15 ; 17)$ positive cases $[3,4]$. Therefore, three distinct types of $P M L-R A R \alpha$ transcripts can be generated in different patients.

The different domains of PML protein retained in the $P M L-R A R \alpha$ isoforms can cause different clinical courses that 


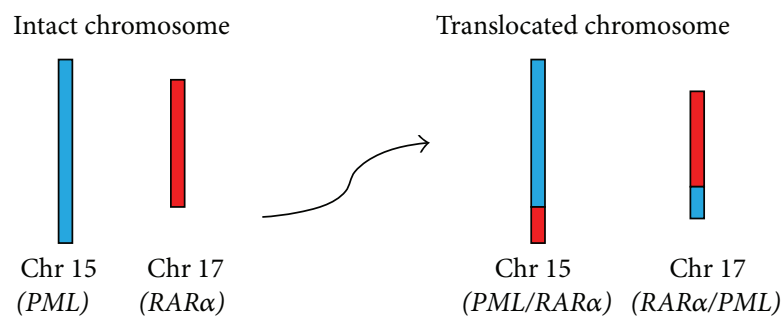

FIGURE 1: Chromosomal reciprocal translocation of the 15 th and 17 th chromosomes.

could affect function and therapeutic responsiveness [5]. Our previous study in Hospital Universiti Sains Malaysia (HUSM) had found that the survival rate was $100 \%$ and $33.3 \%$ for L/Vforms and S-forms, respectively $(P<0.005)$. Median survival for S-form was 19.9 months while all patients with $\mathrm{L} / \mathrm{V}$-form have survived to date [6].

Recent studies have reported that microRNAs (miRNAs) also play critical roles in the pathogenesis of APL, since miRNAs with oncogenic and tumour suppressor activity have been identified [7-11]. Discovery of miRNAs has opened up the possible application in molecular diagnostics and markers for follow up in patients with APL.

In this review, we will briefly discuss the potential roles of miRNAs as biomarkers in APL.

\section{MicroRNAs and Haematological Malignancies}

There is a large volume of published studies describing the potential role of miRNAs in haematological malignancies. miRNAs are small, noncoding RNA ( 22 nucleotides in size) that can regulate many cellular functions, including cell development, proliferation, differentiation, apoptosis, and haematopoiesis $[12,13]$. miRNAs control gene expression at the posttranscriptional level by degrading or repressing target messenger RNAs (mRNAs) [14]. The involvement of miRNAs in cancer has been proven through scientific studies that are growing rapidly. miRNAs have been proposed to contribute to oncogenesis because they can function either as tumour suppressors or oncogenes (Figure 2) [14]. The major general causes of altered miRNAs expression in cancer are genetic alterations [15], epigenetic mechanisms [16], deregulation of miRNAs, mutation in miRNA biogenesis pathway, and mechanism for escape from repression by miRNAs. Abnormal expression of miRNAs has been found in both solid [1723] and haematopoietic tumours [7, 9, 24-27] by various genome-wide techniques.

The first miRNAs reported to have important roles in cancer were miR-15 and miR-16 [28]. Both miRNAs are located at chromosome 13q14, a region deleted in most cases of B cell (B-CLL). MiR-15 and miR-16 were found downregulated in the majority of chronic lymphocytic leukaemia (CLL) cases. This finding was supported by other findings that found that miR-15 and miR-16 were also involved in the pathogenesis of solid cancer such as pituitary adenomas, non-small-cell lung cancer, and prostate cancer [29-31]. The targeted genes that were regulated by miR-15 and miR-16 are Bcl2, CCND1, and $W N T 3 A$. A recent study also found that both miRNAs have a direct feedback on TP53 in CLL [32].

Later, the first miRNA that has a role in myelopoiesis, miR-223, was discovered by Fazi et al. [33]. MiR-223 is regulated by $C E B P a$ transcription factor and is shown to target $M E F 2 c$ transcription factor, a known promoter of myeloid progenitor differentiation.

Other miRNAs are now identified to be involved in the pathogenesis of haematological malignancies. For example, increased expression of the precursor of miR-155 was detected in pediatric Burkitt lymphoma [34], Hodgkin lymphoma [35], and diffuse large B-cell lymphomas [36]. Recently, miR155 was reported to play a unique role in myeloid leukemogenesis [37]. It has been suggested that miR-155 is responsible for the oncogenic function of BIC gene [36]. The elevated levels of miR-155 have also been identified in leukocytes in association with an inflammatory response [38].

A study was carried out by Feng et al. [39] to identify a group of miRNAs that were differentially expressed between exosomes and K562 cells. Exosomes are small membrane vesicles ranging from 40 to $100 \mathrm{~nm}$ in size that carry proteins, RNAs, and microRNAs [40]. The functional role of exosomes in disease initiation and progression had been described in many studies. Tumor cells have been shown to secrete exosomes, and these exosomes can carry the genomic and proteomic signatures characteristic of the tumor cells. It is suggested that exosomes may be involved in cancer cellto-cell communication. Their findings demonstrated that 49 miRNAs were upregulated in K562 cell derived exosomes as compared to K562 cells (Table 1). These finding suggested that miRNAs in exosomes may promote the development of chronic myeloid leukaemia (CML).

MiR-17-92 were found to be downregulated in B-cell lymphoma [41]. This miRNAs cluster was demonstrated to play a key role in lymphomagenesis. Some of their targeted genes (E2F1, E2F3, Pten, AML1, Bim, AIB1, TGFBRII, Tsp1, CTGF, and p130) have been documented to have important roles in hematopoiesis, apoptosis, cell-cycle progression, and angiogenesis, and some have been associated with tumorigenesis [41-45].

Expression studies of miRNAs in AML have shown that miRNA has an important role in haematopoietic development and that expression of miR-181a in particular correlates with morphological subclass [46]. In this study, three other miRNAs, miR-10a, miR-10b, and miR-196a-1, showed a clear correlation with HOX gene expression. Previously, HOX gene expression was reported to be upregulated in leukaemias with a normal karyotype [47].

Subsequent study conducted on miRNAs in AML found that the distinctive pattern of miRNAs can classify the subtypes of AML [27]. For example, miR-127, miR-154, miR-154*, miR-299, miR-323, miR-368, and miR-370 were upregulated in APL (AML with translocation $\mathrm{t}(15 ; 17)$ ) as compared to other subtypes.

All findings suggested that miRNAs may play important roles in haematological malignancies. 


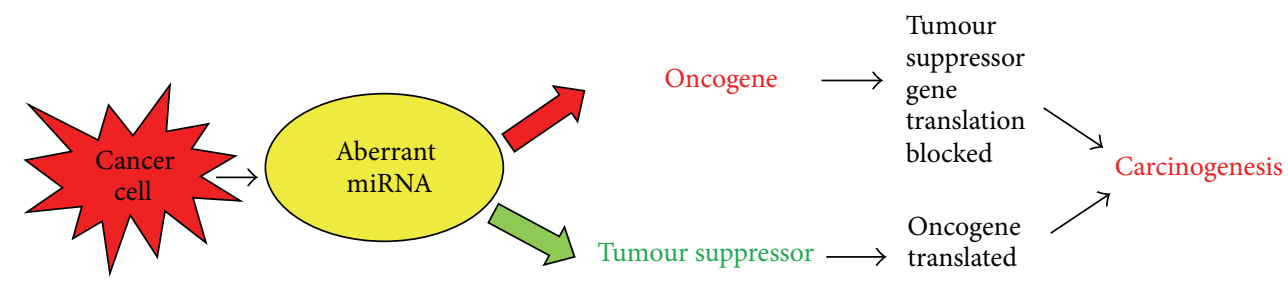

FIgURE 2: MicroRNAs as tumour suppressor and oncogene.

\section{MicroRNAs as Biomarkers in APL}

Acute promyelocytic leukaemia (APL) is a highly curable malignancy. However, some patients will die early at presentation or shortly after initiation of treatment $[48,49]$. Recent studies reported that miRNAs may also play crucial roles in the pathogenesis of APL. Many studies have been conducted to identify possible markers that can be used as therapeutic targets in the management of APL patients.

It is well documented that miRNAs play important roles in cell differentiation by acting as translational inhibitors of specific target genes. The first miRNA reported to be related to APL was miR-223 [50]. This miRNA is specifically expressed in myeloid cells. Two transcriptional factors were identified to be responsible for the differential expression of this miRNA during granulopoiesis: CCAAT/enhancerbinding protein- $\alpha(\mathrm{C} / \mathrm{EBP} \alpha)$ and nuclear factor I-A (NFI-A). The two transcription factors compete for binding to the promoter of miR-223. Although NFI-A maintains miR-223 at low levels, its replacement by $\mathrm{C} / \mathrm{EBP} \alpha$ following retinoic acid differentiation upregulates miR-223 [33]. These findings indicate that miR-223 plays a crucial role during granulopoiesis and clinical response to retinoic acid.

Another study was conducted to further investigate the involvement of miRNAs in the pathogenesis of APL. They reported a group of miRNAs; miR-15a, miR-15b, miR-161, let-7a-3, let-7c, let-7d, miR-223, miR-342, and miR-107 were upregulated and miR-181b was downregulated in APL patients as well as APL cell lines when treated with ATRA [9]. The findings observed in this study mirror those of the previous study that miR-223 was induced significantly upon treatment with ATRA [50]. Interestingly, they found that miR-107, which is predicted to target NFI-A, was upregulated as well after ATRA treatment. Besides, the activation of let7a and miR-15a/miR-16-1 was shown to correlate with the downregulation of RAS and $B c l 2$ genes, respectively. Their findings highlighted the potential of miRNA profiling in the diagnosis of APL.

The contribution of miRNAs in the pathogenesis of APL becomes more interesting to study where a group of miRNAs targeted by PML-RARA has been reported [10]. MiR-15b, miR-223, and miR-342 were found to be upregulated in APL cell lines treated with ATRA whereas the miR-181a and miR$181 \mathrm{~b}$ were downregulated in untreated cell lines. In addition, the let-7a, let-7c, and let-7d miRNAs were also found to be upregulated by ATRA in APL cells and might be repressed by $P M L-R A R A$. The miRNAs repressed by $P M L-R A R A$ are also implicated in the control of crucial pathways linked to
TABLE 1: Differentially expressed miRNAs between exosomes and K562 cells.

\begin{tabular}{lcc}
\hline \multicolumn{2}{c}{ miRNA } & Reference \\
\hline hsa-miR-1908 & Hcmv-miR-UL148D \\
Hsa-miR-30c-1-3p & hsa-miR-943 \\
Hsa-miR-711 & hsa-miR-525-5p \\
Hsa-miR-744-5p & hsa-miR-629-5p \\
Has-miR-4285 & hsa-miR-625-3p \\
Hsa-miR-665 & hsa-miR-107 \\
Hsa-miRPlus-C1087 & hsa-miR-1827 \\
Hsa-miR-423-5p & hsa-miR-483-3p \\
Hsa-miR-3686 & Hsv2-miR-H7-3p \\
Hsa-miR-149-3p & hsa-miR-299-5p \\
Kshv-miR-K12-3 & hsa-let-7e-p \\
Hsv2-miR-H10 & hsa-miR-877-3p \\
Hsa-miR-516b-5p & hsa-miR-1249 \\
Sv40-miR-S1-5p & hsa-miR-3915 \\
Hsa-miR-3201 & hsa-miR-3676-3p \\
Hsa-miR-638 & hsa-miR-1973 \\
hsa-miR-877-5p & hsa-miR-3646 \\
hsa-miR-3611 & hsa-miR-298 \\
hsa-miR-519e-5p & \\
Hsv2-miR-H25 & \\
Ebv-miR-BHRF1-2 & \\
hsa-miR-652-5p & \\
hsa-miR-585 & \\
hsa-miR-4279 & \\
hsa-miR-518b & \\
Hcmv-miR-US25-1* & \\
hsa-miR-4268 & \\
Hsv2-miRH7-5p & \\
hsa-miR-612 & \\
hsa-miR-4290 & \\
hsa-miR-498 & \\
\hline
\end{tabular}

leukemogenesis. For instance, the expression of $H O X B 8$ gene seemed to be controlled by miRNAs potentially repressed by PML-RARA [10].

In APL cell line, miR-181b was proven to regulate APL cell proliferation and apoptosis through targeting MLK2 [25]. $M L K 2$ is a component of the mitogen activated protein kinase (MAPK) pathway which regulates diverse processes, ranging from proliferation and differentiation to apoptosis. 
The MAPK pathway phosphorylates numerous proteins, including transcription factors, cytoskeletal proteins, kinases, and other enzymes, and greatly influences gene expression, metabolism, cell division, cell morphology, and cell survival $[51,52]$.

MiR-181b was found to be upregulated in APL patients as compared to AML patient samples with normal karyotype. In addition, the miR-181b expression was downregulated in APL patient samples after ATRA treatment. Functional study had shown a decreased level of RSSF1A protein in NB4 cell line that was transfected with miR-181b. However, these data were unpublished data [8]. Consistent with this study, this miRNA was also found to be upregulated in gastric cancer cells and primary gastric cancer tissue [53]. MiR-181b maybe suggested as a potential diagnostic biomarker for both cancers.

Upregulation of miR-125b was shown to contribute to leukemogenesis and increased drug resistance in paediatric acute promyelocytic leukaemia patients by targeting Bakl gene [26]. In addition, they found that miR-125b was exceptionally highly expressed in the pediatric APL subtype compared with other AML subtypes. Thus, miR-125b expression should be further examined on a large scale with adult APL patients. Overexpression of miR-125b was able to block myeloid differentiation by targeting $C B F B$, prevent apoptosis through downregulation of multiple genes involved in $p 53$ pathway, and support cytokine independent proliferation of both mouse and human myeloid cell lines (NB4 and HL60) by targeting ABTB1 [54].

A study conducted on a large cohort of AML showed that unique miRNAs expression profiles were present in APL cohort [27]. They found upregulation of a subset of miRNAs located in the human 14q32 imprinted domain. The set included miR-127, miR-154, miR-154*, miR-299, miR-323, miR-368, and miR-370. This study suggested that miRNAs expression profiles can be used to subclassify cancer. In addition, miRNAs may play an important role in the molecular pathogenesis of AML.

Other documented miRNAs involved in the pathogenesis of APL were miR-143 and miR-145. Downregulation of miR-143 and miR-145 was observed in two APL patients who received oral ATRA [7]. This result was obtained by comparing the expression level of miR-143 and miR-145 in total RNA samples taken at diagnosis and day 6 following ATRA therapy. This finding was further validated by qRTPCR.

Recently, inhibition of miR-92a using locked nucleic acid (LNA) analysis was reported to induce apoptosis and inhibit cell proliferation in human APL [11]. Western blot analysis of p63 protein also revealed that miR-92a inhibition resulted in $\mathrm{p} 63$ expression; hence activation of cellular pathways which are normally controlled by $\mathrm{p} 63$ protein was retrieved. MiR-92a was found downregulated in some other diseases including acute leukaemia [55] and multiple myeloma [56].

These findings further support that one miRNA may regulate hundreds or thousands of target genes and one target gene may, respectively, be regulated by several miRNAs. In addition, diverse techniques performed to establish expression profiles of miRNA show unique miRNA signatures that are relevant to the pathogenesis, diagnosis, and prognosis of
APL. Importantly, miRNAs may open a new path for targeted treatments of APL.

\section{Future Perspective}

The potential value of using miRNAs as biomarkers in APL has been shown by numerous promising data. It is based on the fact that changes in miRNA expression profiles have been reported during ATRA treatment and the inhibition of some miRNAs in APL cell lines also showed the alteration in gene expression pattern. Despite strong evidence supporting the potential value of miRNAs as biomarkers, several issues need to be addressed to ensure that the results obtained are consistent. For example, some results showed low correlation obtained from different platforms or even from the same platform using different extraction kits. Detection and quantification of miRNA expression should be robust, rapid, accurate, reproducible, and inexpensive. This is significantly important to make sure that accurate results can be obtained. The discovery of miRNAs as potential diagnostic and prognostic biomarkers will open a new era in the management of APL.

\section{Conclusion}

We believe that this paper will provide new information and better understanding to gain insight into the relationship between various miRNAs signature involved in APL. We conclude that miRNAs have potential contributions in APL and may therefore provide novel biomarkers and therapeutic targets in the management of APL, resulting in a better prognostic outlook for patients diagnosed with this disease in the future.

\section{Conflict of Interests}

The authors declare that there is no conflict of interests regarding the publication of this paper.

\section{Acknowledgment}

Grant support was from Research University Grant (1001/ PPSP/812105), Universiti Sains Malaysia.

\section{References}

[1] J. W. Vardiman, J. Thiele, D. A. Arber et al., "The 2008 revision of the World Health Organization (WHO) classification of myeloid neoplasms and acute leukemia: rationale and important changes," Blood, vol. 114, no. 5, pp. 937-951, 2009.

[2] B.-W. Gu, H. Xiong, Y. Zhou et al., "Variant-type PML-RAR $\alpha$ fusion transcript in acute promyelocytic leukemia: use of a cryptic coding sequence from intron 2 of the RAR $\alpha$ gene and identification of a new clinical subtype resistant to retinoic acid therapy," Proceedings of the National Academy of Sciences of the United States of America, vol. 99, no. 11, pp. 7640-7645, 2002.

[3] P. C. Choppa, J. Gomez, H. G. Vall, M. Owens, H. Rappaport, and J. R. Lopategui, "A novel method for the detection, quantitation, and breakpoint cluster region determination of 
$\mathrm{t}(15 ; 17)$ fusion transcripts using a one-step real-time multiplex RT-PCR," American Journal of Clinical Pathology, vol. 119, no. 1, pp. 137-144, 2003.

[4] T. Chatterjee, S. Gupta, S. Sharma, and P. Ganguli, "Distribution of different PML/RAR $\alpha$ bcr isoforms in Indian acute promyelocytic leukemia (APL) patients and clinicohematological correlation," Mediterranean Journal of Hematology and Infectious Diseases, vol. 6, no. 1, Article ID e2014004, 2014.

[5] F. Lo-Coco, M. Breccia, and D. Diverio, "The importance of molecular monitoring in acute promyelocytic leukaemia," Best Practice and Research: Clinical Haematology, vol. 16, no. 3, pp. 503-520, 2003.

[6] B. A. Hassan, A. D. Abdullah, A. Husin et al., "Characterizing PML/RARA isoforms of Acute Promyelocytic Leukemia (APL) in Malay patients," Bangladesh Journal of Medical Science, vol. 13, no. 3, pp. 311-315, 2014.

[7] J. Batliner, E. Buehrer, M. F. Fey, and M. P. Tschan, "Inhibition of the miR-143/145 cluster attenuated neutrophil differentiation of APL cells," Leukemia Research, vol. 36, no. 2, pp. 237-240, 2012.

[8] D. Braeuer-Hartmann, J.-U. Hartmann, D. Gerloff et al., "PML/RAR $\alpha$-regulated microRNA 181b targets the tumor suppressor RASSF1A in acute promyelocytic leukemia," $A S H$ Annual Meeting Abstract, vol. 118, no. 21, p. 1361, 2011.

[9] R. Garzon, F. Pichiorri, T. Palumbo et al., "MicroRNA gene expression during retinoic acid-induced differentiation of human acute promyelocytic leukemia," Oncogene, vol. 26, no. 28, pp. 4148-4157, 2007.

[10] A. Saumet, G. Vetter, M. Bouttier et al., "Transcriptional repression of microRNA genes by PML-RARA increases expression of key cancer proteins in acute promyelocytic leukemia," Blood, vol. 113, no. 2, pp. 412-421, 2009.

[11] M. Sharifi, R. Salehi, Y. Gheisari, and M. Kazemi, "Inhibition of microRNA miR-92a induces apoptosis and inhibits cell proliferation in human acute promyelocytic leukemia through modulation of p63 expression," Molecular Biology Reports, vol. 41, no. 5, pp. 2799-10, 2014.

[12] R. I. Gregory and R. Shiekhattar, "MicroRNA biogenesis and cancer," Cancer Research, vol. 65, no. 9, pp. 3509-3512, 2005.

[13] D. P. Bartel, "MicroRNAs: genomics, biogenesis, mechanism, and function," Cell, vol. 116, no. 2, pp. 281-297, 2004.

[14] C.-Z. Chen, "MicroRNAs as oncogenes and tumor suppressors," The New England Journal of Medicine, vol. 353, no. 17, pp. 17681771, 2005.

[15] A. Laganà, F. Russo, C. Sismeiro, R. Giugno, A. Pulvirenti, and A. Ferro, "Variability in the incidence of miRNAs and genes in fragile sites and the role of repeats and $\mathrm{CpG}$ islands in the distribution of genetic material," PLoS ONE, vol. 5, no. 6, Article ID el1166, 2010.

[16] P. Lopez-Serra and M. Esteller, "DNA methylation-associated silencing of tumor-suppressor microRNAs in cancer," Oncogene, vol. 31, no. 13, pp. 1609-1622, 2012.

[17] S. Akhavantabasi, A. Sapmaz, S. Tuna, and A. E. Erson-Bensan, "MiR-125b targets ARID3B in breast cancer cells," Cell Structure and Function, vol. 37, no. 1, pp. 27-38, 2012.

[18] N.-S. A. Mutalib, C. Yoke-Kqueen, S. A. Rahman, S. M. Sidik, A. S. M. Singh, and L. Learn-Han, "Differential microRNA expression and identification of putative miRNA targets and pathways in head and neck cancers," International Journal of Molecular Medicine, vol. 28, no. 3, pp. 327-336, 2011.

[19] Y. Saito, G. Liang, G. Egger et al., "Specific activation of microRNA-127 with downregulation of the proto-oncogene
BCL6 by chromatin-modifying drugs in human cancer cells," Cancer Cell, vol. 9, no. 6, pp. 435-443, 2006.

[20] J. Takamizawa, H. Konishi, K. Yanagisawa et al., "Reduced expression of the let-7 microRNAs in human lung cancers in association with shortened postoperative survival," Cancer Research, vol. 64, no. 11, pp. 3753-3756, 2004.

[21] M. Weber, M. B. Baker, R. S. Patel, A. A. Quyyumi, G. Bao, and C. D. Searles, "MicroRNA expression profile in CAD patients and the impact of ACEI/ARB," Cardiology Research and Practice, vol. 2011, Article ID 532915, 5 pages, 2011.

[22] W. Wu, J. Yang, X. Feng et al., "MicroRNA-32 (miR-32) regulates phosphatase and tensin homologue (PTEN) expression and promotes growth, migration, and invasion in colorectal carcinoma cells," Molecular Cancer, vol. 12, no. 1, article 30, 2013.

[23] S. Volinia, G. A. Calin, C.-G. Liu et al., "A microRNA expression signature of human solid tumors defines cancer gene targets," Proceedings of the National Academy of Sciences of the United States of America, vol. 103, no. 7, pp. 2257-2261, 2006.

[24] D. L. Zanette, F. Rivadavia, G. A. Molfetta et al., "miRNA expression profiles in chronic lymphocytic and acute lymphocytic leukemia," Brazilian Journal of Medical and Biological Research, vol. 40, no. 11, pp. 1435-1440, 2007.

[25] H. Chen, Q. Chen, M. Fang, and Y. Mi, "microRNA-181b targets MLK2 in HL-60 cells," Science China Life Sciences, vol. 53, no. 1, pp. 101-106, 2010.

[26] H. Zhang, X.-Q. Luo, D.-D. Feng et al., "Upregulation of microRNA-125b contributes to leukemogenesis and increases drug resistance in pediatric acute promyelocytic leukemia," Molecular Cancer, vol. 10, article 108, 2011.

[27] A. Dixon-McIver, P. East, C. A. Mein et al., "Distinctive patterns of microRNA expression associated with karyotype in acute myeloid leukaemia," PLoS ONE, vol. 3, no. 5, Article ID e2141, 2008.

[28] G. A. Calin, C. D. Dumitru, M. Shimizu et al., "Frequent deletions and down-regulation of micro-RNA genes miR15 and miR16 at 13q14 in chronic lymphocytic leukemia," Proceedings of the National Academy of Sciences of the United States of America, vol. 99, no. 24, pp. 15524-15529, 2002.

[29] D. Bonci, V. Coppola, M. Musumeci et al., "The miR-15amiR-16-1 cluster controls prostate cancer by targeting multiple oncogenic activities," Nature Medicine, vol. 14, no. 11, pp. 1271$1277,2008$.

[30] N. Bandi, S. Zbinden, M. Gugger et al., "miR-15a and miR-16 are implicated in cell cycle regulation in a Rb-dependent manner and are frequently deleted or down-regulated in non-small cell lung cancer," Cancer Research, vol. 69, no. 13, pp. 5553-5559, 2009.

[31] A. Bottoni, D. Piccin, F. Tagliati, A. Luchin, M. C. Zatelli, and E. C. D. Uberti, "miR-15a and miR-16-1 down-regulation in pituitary adenomas," Journal of Cellular Physiology, vol. 204, no. 1, pp. 280-285, 2005.

[32] M. Fabbri, A. Bottoni, M. Shimizu et al., "Association of a microRNA/TP53 feedback circuitry with pathogenesis and outcome of b-cell chronic lymphocytic leukemia," JAMAJournal of the American Medical Association, vol. 305, no. 1, pp. 59-67, 2011.

[33] F. Fazi, A. Rosa, A. Fatica et al., "A minicircuitry comprised of microRNA-223 and transcription factors NFI-A and C/EBP $\alpha$ regulates human granulopoiesis," Cell, vol. 123, no. 5, pp. 819831, 2005.

[34] M. Metzler, M. Wilda, K. Busch, S. Viehmann, and A. Borkhardt, "High expression of precursor microRNA-155/BIC 
RNA in children with Burkitt lymphoma," Genes Chromosomes and Cancer, vol. 39, no. 2, pp. 167-169, 2004.

[35] J. Kluiver, S. Poppema, D. de Jong et al., "BIC and miR-155 are highly expressed in Hodgkin, primary mediastinal and diffuse large B cell lymphomas," Journal of Pathology, vol. 207, no. 2, pp. 243-249, 2005.

[36] P. S. Eis, W. Tam, L. Sun et al., "Accumulation of miR-155 and BIC RNA in human B cell lymphomas," Proceedings of the National Academy of Sciences of the United States of America, vol. 102, no. 10, pp. 3627-3632, 2005.

[37] G. Marcucci, K. S. Maharry, K. H. Metzeler et al., "Clinical role of microRNAs in cytogenetically normal acute myeloid leukemia: miR-155 upregulation independently identifies highrisk patients," Journal of Clinical Oncology, vol. 31, no. 17, pp. 2086-2093, 2013.

[38] R. M. O’Connell, K. D. Taganov, M. P. Boldin, G. Cheng, and D. Baltimore, "MicroRNA-155 is induced during the macrophage inflammatory response," Proceedings of the National Academy of Sciences of the United States of America, vol. 104, no. 5, pp. 16041609, 2007.

[39] D.-Q. Feng, B. Huang, J. Li et al., "Selective miRNA expression profile in chronic myeloid leukemia K562 cell-derived exosomes," Asian Pacific Journal of Cancer Prevention, vol. 14, no. 12, pp. 7501-7508, 2013.

[40] M. C. Henderson and D. O. Azorsa, "The genomic and proteomic content of cancer cell-derived exosomes," Frontiers in Oncology, vol. 2, article 38, Article ID 00038, 2012.

[41] M. Inomata, H. Tagawa, Y.-M. Guo, Y. Kameoka, N. Takahashi, and K. Sawada, "MicroRNA-17-92 down-regulates expression of distinct targets in different B-cell lymphoma subtypes," Blood, vol. 113, no. 2, pp. 396-402, 2009.

[42] H. Tagawa, K. Karube, S. Tsuzuki, K. Ohshima, and M. Seto, "Synergistic action of the microRNA-17 polycistron and Myc in aggressive cancer development," Cancer Science, vol. 98, no. 9, pp. 1482-1490, 2007.

[43] K. A. O’Donnell, E. A. Wentzel, K. I. Zeller, C. V. Dang, and J. T. Mendell, "c-Myc-regulated microRNAs modulate E2F1 expression," Nature, vol. 435, no. 7043, pp. 839-843, 2005.

[44] L. Fontana, E. Pelosi, P. Greco et al., "MicroRNAs 17-5p-20a106a control monocytopoiesis through AML1 targeting and MCSF receptor upregulation," Nature Cell Biology, vol. 9, no. 7, pp. 775-787, 2007.

[45] Q. Wang, C. L. Yan, J. Wang et al., "miR-17-92 cluster accelerates adipocyte differentiation by negatively regulating tumorsuppressor Rb2/p130," Proceedings of the National Academy of Sciences of the United States of America, vol. 105, no. 8, pp. 28892894, 2008.

[46] S. Debernardi, S. Skoulakis, G. Molloy, T. Chaplin, A. DixonMcIver, and B. D. Young, "MicroRNA miR-181a correlates with morphological sub-class of acute myeloid leukaemia and the expression of its target genes in global genome-wide analysis," Leukemia, vol. 21, no. 5, pp. 912-916, 2007.

[47] S. Debernardi, D. M. Lillington, T. Chaplin et al., "Genomewide analysis of acute myeloid leukemia with normal karyotype reveals a unique pattern of homeobox gene expression distinct from those with translocation-mediated fusion events," Genes Chromosomes and Cancer, vol. 37, no. 2, pp. 149-158, 2003.

[48] S. Castaigne, C. Chomienne, M. T. Daniel et al., "All-trans retinoic acid as a differentiation therapy for acute promyelocytic leukemia. I. Clinical results," Blood, vol. 76, no. 9, pp. 1704-1709, 1990.
[49] G. Mann, D. Reinhardt, J. Ritter et al., “Treatment with all-trans retinoic acid in acute promyelocytic leukemia reduces early deaths in children," Annals of Hematology, vol. 80, no. 7, pp. 417422, 2001.

[50] C. Nervi, F. Fazi, A. Rosa, A. Fatica, and I. Bozzoni, "Emerging role for microRNAs in acute promyelocytic leukemia," Current Topics in Microbiology and Immunology, vol. 313, pp. 73-84, 2007.

[51] W. Wu, T. Pew, M. Zou, D. Pang, and S. D. Conzen, "Glucocorticoid receptor-induced MAPK phosphatase-1 (MPK-1) expression inhibits paclitaxel-associated MAPK activation and contributes to breast cancer cell survival," Journal of Biological Chemistry, vol. 280, no. 6, pp. 4117-4124, 2005.

[52] J. M. Kim, J. M. White, A. S. Shaw, and B. P. Sleckman, "MAPK p38 $\alpha$ is dispensable for lymphocyte development and proliferation," The Journal of Immunology, vol. 174, no. 3, pp. 1239-1244, 2005.

[53] J.-X. Guo, Q.-S. Tao, P.-R. Lou, X.-C. Chen, J. Chen, and G.-B. Yuan, "miR-181b as a potential molecular target for anticancer therapy of gastric neoplasms," Asian Pacific Journal of Cancer Prevention, vol. 13, no. 5, pp. 2263-2267, 2012.

[54] M. Bousquet, D. Nguyen, C. Chen, L. Shields, and H. F. Lodish, "MicroRNA-125b transforms myeloid cell lines by repressing multiple mRNA," Haematologica, vol. 97, no. 11, pp. 1713-1721, 2012.

[55] M. Tanaka, K. Oikawa, M. Takanashi et al., "Down-regulation of miR-92 in human plasma is a novel marker for acute leukemia patients," PLoS ONE, vol. 4, no. 5, Article ID e5532, 2009.

[56] S. Yoshizawa, J. H. Ohyashiki, M. Ohyashiki et al., "Downregulated plasma miR-92a levels have clinical impact on multiple myeloma and related disorders," Blood Cancer Journal, vol. 2, no. 1, article e53, 2012. 

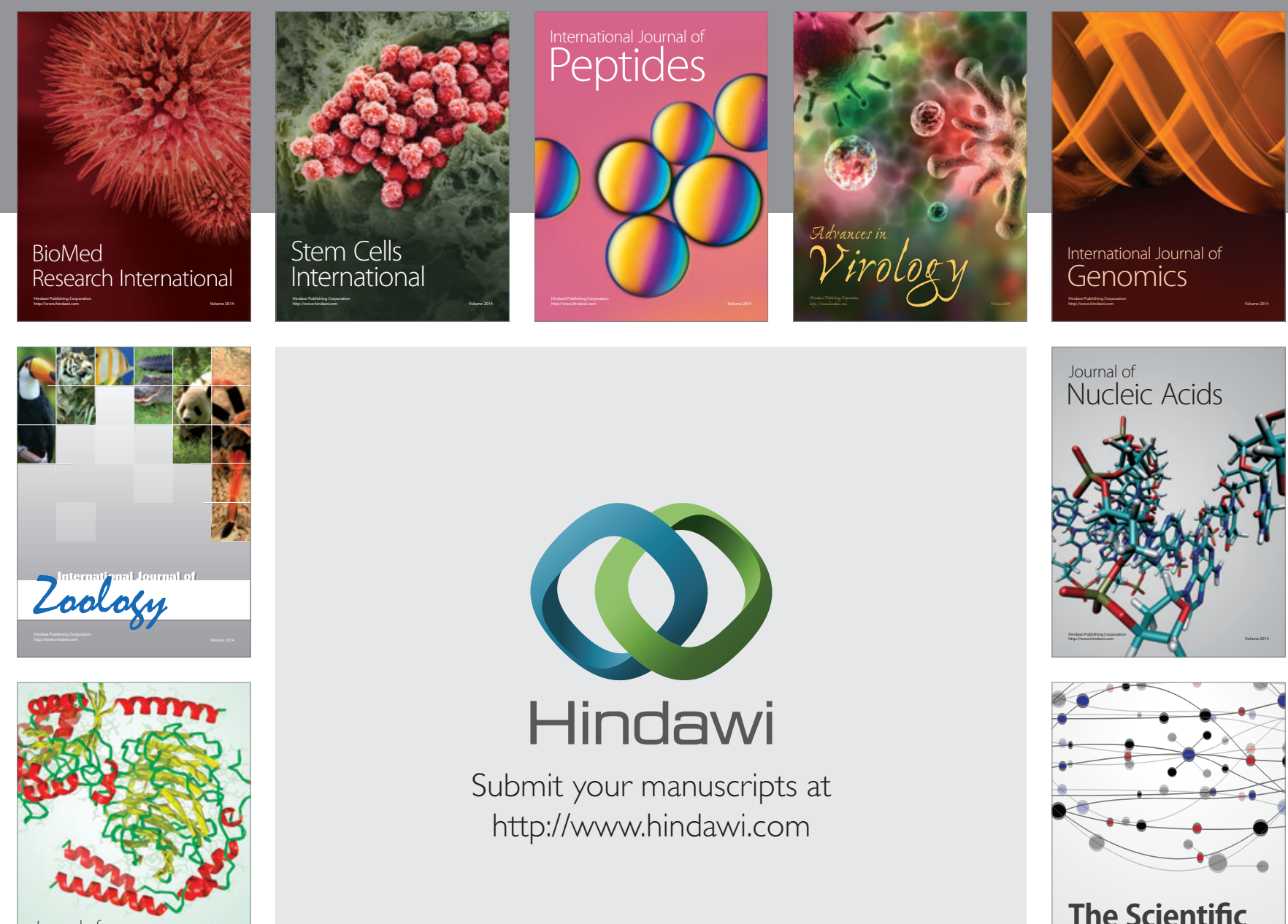

Submit your manuscripts at

http://www.hindawi.com

Journal of
Signal Transduction
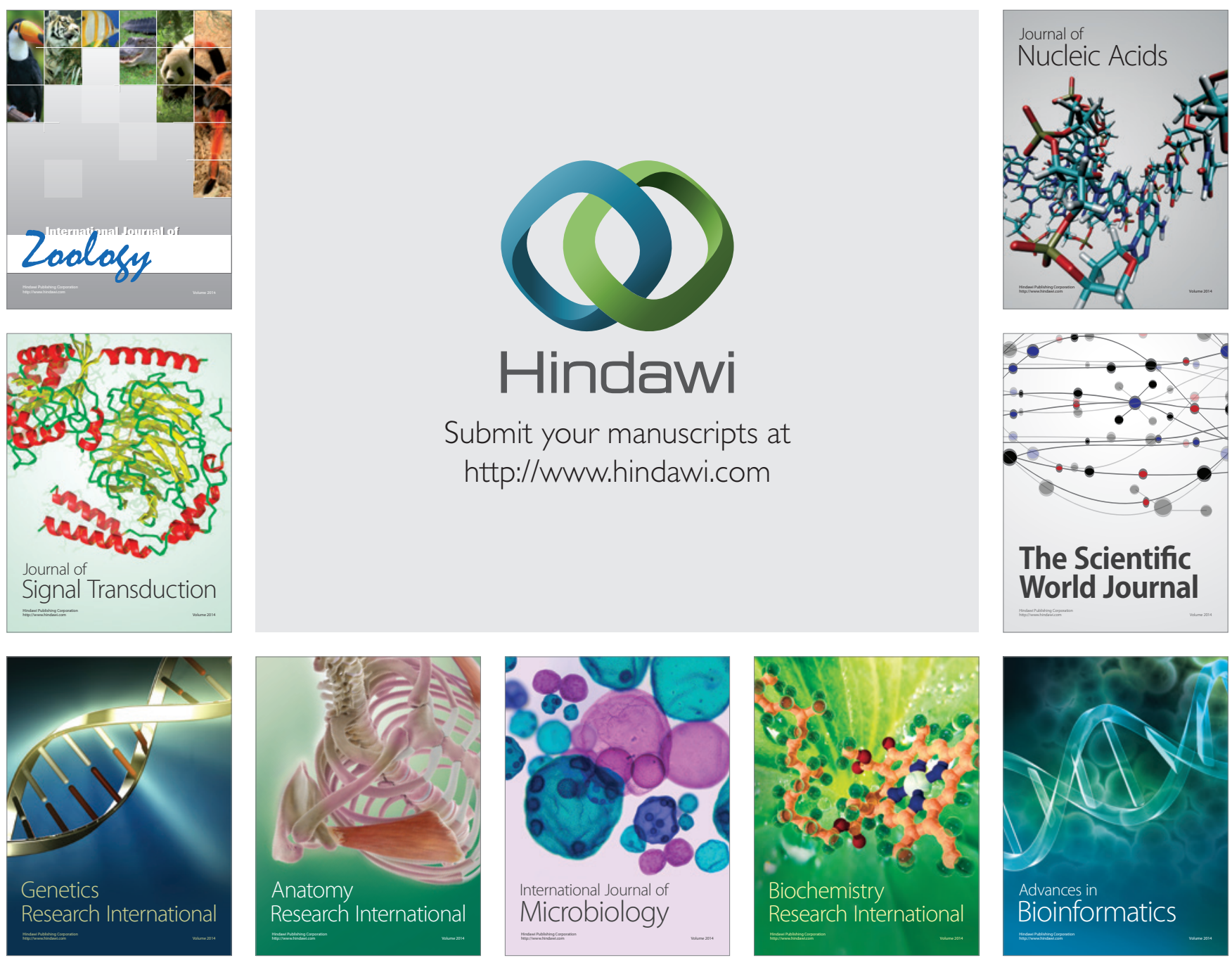

The Scientific World Journal
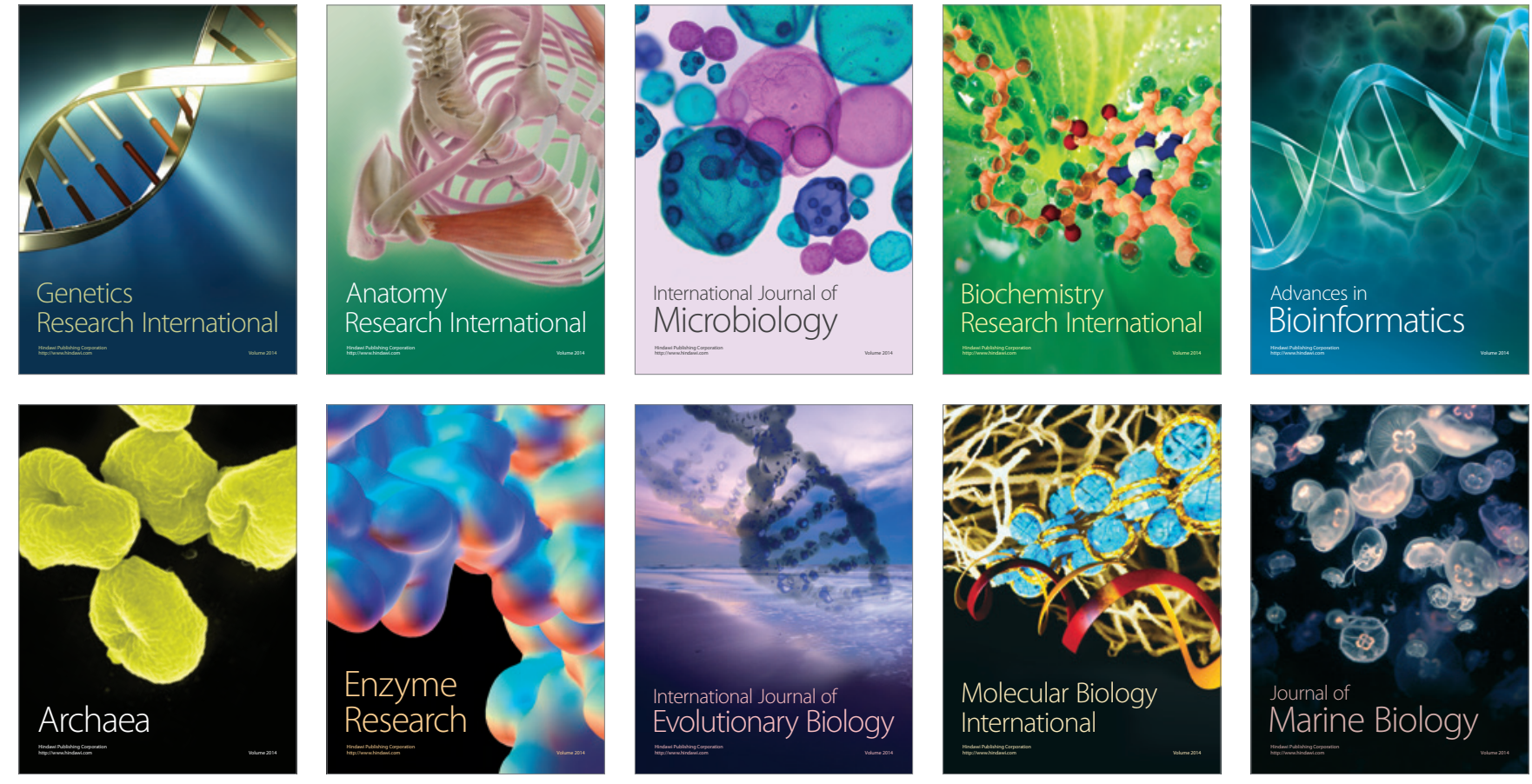